\title{
Monte Carlo techniques for time-dependent radiative transfer in 3-D supernovae
}

\author{
L. B. Lucy \\ Astrophysics Group, Blackett Laboratory, Imperial College London, Prince Consort Road, London SW7 2AZ, UK \\ e-mail: 1.1ucy@imperial.ac.uk \\ Received 13 July 2004 / Accepted 7 September 2004

\begin{abstract}
Monte Carlo techniques based on indivisible energy packets are described for computing light curves and spectra for 3-D supernovae. The radiative transfer is time-dependent and includes all effects of $O(v / c)$. Monte Carlo quantization of a single energy packet comprising $\gamma$-ray photons representing one line from either the ${ }^{56} \mathrm{Ni}$ or the ${ }^{56} \mathrm{Co}$ decay spectrum. Subsequently, these energy packets propagate through the homologously-expanding ejecta with appropriate changes in the nature of their contained energy as they undergo Compton scatterings and pure absorptions.

The 3-D code is tested by applying it to a spherically-symmetric SN in which the transfer of optical radiation is treated with a grey absorption coefficient. This 1-D problem is separately solved using Castor's co-moving frame moment equations. Satisfactory agreement is obtained.

The Monte Carlo code is a platform onto which more advanced treatments of the interactions of matter and radiation can be added. Some of these have already been developed and tested in previous papers and are summarized here.
\end{abstract} \\ is achieved by discretizing the initial distribution of ${ }^{56} \mathrm{Ni}$ into $\mathcal{N}$ radioactive pellets. Each pellet decays with the emission
}

Key words. stars: supernovae: general - radiative transfer - methods: numerical

\section{Introduction}

In earlier papers, Monte Carlo (MC) methods were used to construct spectral synthesis codes for $\mathrm{SNe}$ in their photospheric phases. Because the emphasis was on coarse analyses of the spectra of newly-discovered $\mathrm{SNe}$, major simplifying approximations were made to minimize computing time and to ensure robustness. Thus, in the first code (Lucy 1987; Mazzali \& Lucy 1993), the SN's atmosphere is spherically-symmetric and homologously expanding, the radiation field is stationary $(c=\infty)$, continuum formation is confined to a sharply-defined lower boundary (Schuster-Schwarzschild approximation), and line formation results from the coherent scattering of this continuum as it propagates through the outer layers. Moreover, the stratifications of temperature, ionization and excitation are derived not from first principles but from formulae that approximate the effects of dilution in the SN's extended atmosphere.

In a subsequent paper (Lucy 1999b), two innovations significantly improved this code. First, coherent scattering was replaced by downward branching as the mechanism of line formation. Secondly, a computed spectrum's sampling errors were greatly reduced by using the formal integral for the emergent intensity instead of simply binning the escaping photon packets.

Although these codes have proved their worth diagnostically, they cannot compute spectra for explosion models, a challenge that must be faced if explosion mechanisms and progenitor scenarios are to be confronted with observed spectra and light curves. Evidently, simplifying assumptions must be reconsidered in planning a more powerful and versatile code.

First, the assumption of stationarity must be abandoned. As Arnett $(1980,1982)$ long ago demonstrated for SNe both of types I and II, the luminosity $L(t)$ at elapsed time $t$ does not in general closely approximate the instantaneous energy deposition rate by $\gamma$-rays emitted in radioactive decays. Accordingly, the time-dependent diffusion of radiant energy through the expanding ejecta must be treated explicitly if we wish to compute the emergent luminosity density $L_{v}$ as a function of time.

Similarly, artificially creating radiant energy by means of a continuum-emitting lower boundary is no longer acceptable. The computational domain must be the complete configuration, not just a "reversing layer"; and continuum formation must be treated explicitly. Escaping radiant energy then derives ultimately from radioactive decays or from the energy content of the ejecta at $t_{1}$, the time at which the output from an explosion calculation is used to initiate the spectral synthesis code.

Removal of the constraint of spherical symmetry is also highly desirable in view of accumulating observational evidence and theoretical arguments implying that most and perhaps all SN explosions are significantly aspherical - see Wheeler (2004) for a recent review.

Of the assumptions in the earlier codes, the only one retained is that of homologous expansion. This requires that the explosion calculation, which of necessity includes gas 
dynamics, be continued until all mass elements are to a good approximation coasting ballistically.

With the assumptions to be dropped identified, the problem is now defined: to solve the time-dependent, 3-D NLTE transfer problem for UVOIR radiation in the homologously expanding ejecta of a SN, given the distribution of mass and composition at an initial time $t_{1}$. Of course, this problem is coupled to a corresponding 3-D transport problem for the $\gamma$-rays emitted by radioactive isotopes. This coupling occurs via the equations describing the deposition and degradation of $\gamma$-ray energy.

In view of the magnitude of this problem, this paper is restricted to describing an exploratory MC code in which the UVOIR radiation's interaction with matter is governed by a grey absorption coefficient. This problem was previously treated by Pinto \& Eastman (2000) in order to explore the sensitivity of type Ia light curves to parameters. Here the aim is to create a software platform onto which more realistic physics can be subsequently added and with which numerical techniques can be tested.

Monte Carlo methods are a natural choice for this problem given the experience with the earlier codes. Moreover, MC methods are commonly favoured for transport phenomena in geometrically-complex configurations with no symmetries. Nevertheless, the conventional approach in which the derivatives in the time-dependent transfer equation (RTE) are approximated by differences has already been partially implemented for 3-D SNe by Höflich (2003). With regard to the application of MC methods, the most advanced work seems to be that of Kasen et al. (2004) who carry out 2-D time-independent calculations to compute the observable characteristics of a type Ia model with a conical hole.

\section{Monte Carlo techniques}

In this section, after some preliminaries, recent developments in MC technique that are relevant for a general spectral synthesis code are summarized. These remarks are not restricted to the grey case considered in this paper.

\subsection{Random numbers}

Random numbers are obtained with a double precision version of the routine ran2 of Press et al. (1992). Such numbers are always denoted by $z$, with each $z$ denoting an independent call to $\operatorname{ran} 2$.

\subsection{Energy packets}

As in earlier codes, the MC quanta are energy packets. In general, these are referred to as $\mathcal{E}$-packets except when specifying the nature of the contained energy (Paper I, Sect. 2). Thus $r$-packets and $\gamma$-packets are monochromatic photon packets of UVOIR radiation and of $\gamma$-rays, respectively. On the other hand, $k$-packets and $i$-packets contain not radiation but thermal kinetic energy and ionization energy, respectively. In addition, a full treatment of radioactive decay and $\gamma$-ray deposition will require consideration of $\mathrm{e}^{-}-$and $\mathrm{e}^{+}$-packets containing non-thermal electrons and positrons, respectively.

\subsection{Discretization}

The $\mathrm{SN}$ is enclosed in a Cartesian grid containing $I^{3}$ identical cubic cells. This grid expands with the ejecta so that each cell contains a fixed parcel of matter. Physical variables do not vary spatially within cells.

The expansion itself is discretized into $N$ time steps. Expansion occurs in instantaneous jumps at times $t_{n}$ separated by a constant value of $\Delta \log t$. During every time inter$\operatorname{val}\left(t_{n}, t_{n+1}\right)$, the density $\rho$ of each cell is held fixed at the value predicted by homologous expansion at time $t_{n+1 / 2}=\sqrt{t_{n} t_{n+1}}$.

Here, and throughout the paper, $t$ is the elapsed time since explosion and is measured in the SN's centre-of-mass rest frame (rf).

With this time-stepping, the calculation is divided into $N$ separate MC experiments, each of which updates the radiation field throughout the grid from time $t_{n}$ to $t_{n+1}$ for $n=1,2, \ldots, N$.

\subsection{Relativistic terms}

Given the modest accuracy with which we can currently treat many of the physical processes in SN ejecta, all terms of $O(v / c)$ in radiative transfer could be neglected except for Doppler frequency shifts, which are essential in line transfer. Nevertheless, looking forward to a time when the relevant cross sections will be known to high precision, terms of $O(v / c)$ are now included.

The basic transfer calculations are carried out in the rf, but transformations to and from the local co-moving frame $(\mathrm{cmf})$ are convenient in treating interactions with matter. The motion of an $\mathcal{E}$-packet is thus given by $\boldsymbol{r}(t)$, its position as a function of time $t$ in the rf Cartesian coordinate system with origin at the SN's centre of mass. When the grid attached to the ejecta expands instantaneously at times $t_{n}$, the coordinates $\boldsymbol{r}$ and direction vectors $\mu$ of the $\mathcal{E}$-packets remain unchanged.

\subsection{Energy conservation}

In the context of this discretization of space and time, energy conservation implies $I^{3} N$ constraints that the solution must satisfy. Thus, for each cell and every time step, the net emissivity of radiant energy per unit mass must be balanced by energy created within the mass element after subtracting the increase $\mathrm{d} U$ in its internal energy and the $p \mathrm{~d} V$ work done by gas pressure.

With conventional transfer techniques, a solution satisfying this huge number of constraints - perhaps $\sim 10^{8}-$ has to be achieved iteratively - and convergence is never rapid. In contrast, if the quanta in a MC calculation are indestructible and indivisible $\mathcal{E}$-packets, local energy conservation is automatically and rigorously obeyed, even if physical variables have not converged to their final values (Lucy 1999a).

To a good approximation, energy conservation can be simplified to the condition of thermal equilibrium by neglecting the gas terms $\mathrm{d} U+p \mathrm{~d} V$ (Arnett 1980; Pinto \& Eastman 2000a). With this simplification, the net emissivity for UVOIR radiation in the cmf is equal to the rate of energy deposition by $\gamma$-rays. 
Thermal equilibrium is rigorously obeyed by the MC calculation because the effective cmf emissivity $j_{\text {eff }}^{\prime}(v)$ implied by using indivisible $\mathcal{E}$-packets is subject to the integral constraint

$4 \pi \int j_{\text {eff }}^{\prime}(v) \mathrm{d} v=4 \pi \int k_{v}^{\prime} J_{v}^{\prime} \mathrm{d} v+\mathcal{H}_{\gamma}^{\prime}$

where $k_{v}^{\prime}$ is the cmf absorption coefficient per unit volume, $J_{v}^{\prime}$ is the cmf mean intensity, and $\mathcal{H}_{\gamma}^{\prime}$ is the $\mathrm{cmf} \gamma$-ray energy deposition rate per unit volume. In contrast, when the RTE is solved conventionally, the cmf emissivity $j_{v}^{\prime}$ is given by the fundamental NLTE formulae relating the emission of photons by boundbound (b-b) and free-bound (f-b) transitions to the level populations and the electron temperature. Equation (1) is then only satisfied asymptotically as the iterative procedure converges.

Note that, in contrast to $j_{v}^{\prime}$ derived from first priniples, the MC emissivity $j_{\mathrm{eff}}^{\prime}(v)$ is not defined by mathematical formulae. Rather it is defined operationally by the rules governing the reemission of absorbed $\mathcal{E}$-packets (Papers I, II).

\subsection{Statistical equilibrium}

In addition to the constraints demanded by thermal equilibrium, an even larger number of constraints are required by statistical equilibrium. During each time step and for each cell, the number of excitations of an atomic level must balance the number of de-excitations. With accurate models for the atoms in the multi-species plasma, the total number of levels might be $\sim 10^{3}$. This is therefore the number of constraints per cell per time step implied by statistical equilibrium. Combining this with the earlier estimate of $I^{3} N$, we see that $\sim 10^{11}$ constraints must be satisfied in the course of computing NLTE light curves and spectra of a 3-D SN.

Indivisible $\mathcal{E}$-packets allow the constraint of statistical equilibrium to be incorporated automatically and rigorously, even when excitation and de-excitation rates computed from basic formulae do not balance. This is achieved with the macroatom formalism (Paper I), according to which the rate of de-excitation of an excited state is not determined by its level population but by the rates at which radiative and collisional processes from all other levels populate the level in question, either directly or through the internal transitions of the macroatom.

If the radiation field is computed from the RTE, errors in level populations, such as those present prior to convergence, result in the non-physical creation or destruction of radiant energy. This problem is a consequence of the generality of the RTE equation with the NLTE emissivity $j_{v}^{\prime}$. Because of this generality, the NLTE RTE could be used to follow the timedependent relaxation to statistical equilibrium. In contrast, with the macro-atom formalism, spurious sources or sinks of radiant energy are eliminated. This is achieved by effectively solving a less general transfer equation, namely one that incorporates the constraint of statistical equilibrium and one, therefore, that cannot be used for a relaxation calculation.

A consequence of computing de-excitation rates with the macro-atom machinery is that these rates and the corresponding emissivity $j_{\text {eff }}^{\prime}(v)$ are insensitive to errors in the populations of the emitting levels (Paper I, Sect. 6). In contrast, with the
RTE, errors in level populations translate directly into errors in $j_{v}^{\prime}$ and therefore in the derived radiation field. Evidently, the macro-atom approach is more tolerant of departures from the NLTE solution than is the conventional approach.

As thus far developed, the macro-atom approach imposes the constraint of thermal equilibrium simultaneously with that of statistical equilibrium. Thus when a $k$-packet is created (Paper II, Sect. 4.3.1), it is instantaneously eliminated (Paper II, Sect. 4.3.2). Thus, there is no net energy transfer to or from the thermal pool. Moreover, this holds even if the electron temperature does not imply thermal equilibrium. Note also that if a $k$-packet is created by the de-activation of a macro-atom of one atomic species, it may be eliminated by the activation of a macro-atom of another species.

\subsection{Non-equilibrium}

For $\mathrm{SNe}$ ejecta at late times, the assumptions of ionization and thermal equilibrium break down (Fransson \& Kozma 1993). But this does not preclude the use of indivisible $\mathcal{E}$-packets, since these are fundamentally a means of tracking energy. Departures from equilibrium can be modelled by allowing for the finite life time of a non-radiative $\mathcal{E}$-packet before it converts back into an $r$-packet. Thus a $b-f$ process creates a $k$-packet or an $i$-packet, but neither should be immediately eliminated if the recombination time is not short compared to the elapsed time $t$. Moreover, while awaiting elimination, the $k$-packet's energy declines due to $p \mathrm{~d} V$ work.

If, during such non-equilibrium phases, statistical equilibrium remains a good approximation for the excited levels of each ion separately, then this constraint can be imposed by introducing macro-ions adapting the procedures of Paper I.

The above remarks strongly suggest that non-equilibrium phases can be treated with closely similar techniques. Nevertheless, this must be confirmed with test problems as in Paper I.

\subsection{A-iterations}

The robustness of the emissivities derived with the macroatom formalism suggests that useful predictions of $L_{v}(t)$ may be obtained without converging to the exact NLTE solution. Instead, estimates of the excitation, ionization and temperature in each cell could be derived using the characteristics of the local MC radiation field as in Abbott \& Lucy (1985) and in the previous diagnostic codes (Sect. 1). The accuracy of this approach in computing ionization fractions has been confirmed by Springmann \& Puls (1998) for an O-star wind.

Nevertheless, for definitive results, the NLTE solution must be obtained. Fortunately, the use of indivisible $\mathcal{E}$-packets and the macro-atom formalism facilitate this task. Because the thermal and statistical equilibrium constraints are directly incorporated into the MC calculation, convergence to the NLTE solution can be achieved with geometry-independent $\Lambda$-iterations (Lucy 1999a; Paper II). Thus, by simply repeating the process of bringing matter into thermal and statistical equilibrium with the $\mathrm{MC}$ radiation field and then recomputing the latter, the 
solution converges to the required equilibria. Moreover, convergence is rapid.

With regard to thermal equilibrium, this success with $\Lambda$-iterations was initially demonstrated for a 1-D problem (Lucy 1999a). Recently, the geometric independence of the technique has been demonstrated with its successful application to demanding 3-D problems (Harries et al. 2004; Kurosawa et al. 2004). With regard to thermal and statistical equilibrium, experience is thus far limited to a simple 1-D problem (Paper II).

\subsection{Monte Carlo estimators}

A NLTE calculation requires the radiative rates of excitation, ionization and heating. With the RTE, these are obtained by numerical integration. But computing these quantities in a MC simulation is not so straightforward. The obvious approach is simply to count the relevant events - e.g, photoionizations - in each cell in time $\Delta t$, thereby deriving the rate empirically. This is akin to a physics experiment in which detectors are distributed throughout an apparatus to record events. But, though appealing, this fails to make full use of the MC radiation field. For example, even if no photoionizations occur in a cell, a non-zero ionization rate must surely be derivable if it was traversed by $r$-packets containing ionizing photons.

To derive MC estimators of radiative rates, a summation procedure based on volume elements is preferred (Lucy 1999) to the more obvious choice of reference surfaces. The basic building block from which the required estimators are derived will now be stated in a more general form than in earlier papers (Lucy 1999; Paper II).

If, at a given position and time, $I_{v}(\theta, \phi)$ is the specific intensity at frequency $v$ of a pencil of radiation propagating in direction $(\theta, \phi)$, then $4 \pi I_{v} / c \times \mathrm{d} v \mathrm{~d} \omega$ is the instantaneous energy density of radiation in the frequency interval $(v, v+\mathrm{d} v)$ propagating within solid angle $\mathrm{d} \omega$ about the specified direction. This basic formula allows the $\mathrm{MC}$ radiation field to be converted into the conventional description in terms of $I_{v}$.

If, during $\Delta t$, an $r$-packet of energy $\epsilon_{v}$ with $v \in(v, v+\mathrm{d} v)$ propagates in a cell of volume $V$, and if for a time $\delta t=\delta s / c$ its trajectory is in the solid angle element $\mathrm{d} \omega$, then $\epsilon_{v} \delta s / c$ is the packet's contribution to the cell's time-integrated radiant energy in $\mathrm{d} v \mathrm{~d} \omega$. Accordingly, the volume- and time-averaged estimator of the specific intensity is given by

$I_{v} \mathrm{~d} \nu \mathrm{d} \omega=\frac{1}{4 \pi} \frac{\epsilon_{0}}{\Delta t} \frac{1}{V} \sum_{\mathrm{d} \nu \mathrm{d} \omega} \frac{\epsilon_{v}}{\epsilon_{0}} \delta s$.

Here $\epsilon_{0}$ is the reference value for the energy of the $\mathcal{E}$-packets (Sect. 3.2).

Equation (2), when multiplied by the appropriate weight functions and integrated over $v$ and $\omega$, yields MC estimators for any required property of the radiation field. Estimators of this type were used initially to compute integrated mean intensities and absorption rates in a 1-D non-grey atmosphere in LTE (Lucy 1999a). Similar applications to dusty circumstellar envelopes have been reported by Wolf (2003) and by Niccolini et al. (2003). Estimators for the radiative rates needed for NLTE calculations can also be derived (Paper II).
The class of MC estimators derived from Eq. (2) can be described as optimum and non-parametric. Optimum because they use all the MC information and non-parametric because no assumption is made about the radiation field. These estimators are appropriate when the simulation is large enough that in each $\Delta t$ every cell is traversed by many $\mathcal{E}$-packets. If not, a functional form can be assumed for $I_{v}$ or $J_{v}-$ e.g., a dilute black body - and its parameters estimated from the limited number of trajectories in $V$. This functional form can then be multiplied by the appropriate weight functions to derive an estimate of the required quantity by numerical integration. This second procedure dampens the effects of sampling errors but, insofar as the functional form is inexact, yields biased estimators - i.e., ones that do not converge to their exact values as $\mathcal{N} \rightarrow \infty$, where $\mathcal{N}$ is the number of packets in the simulation.

An application where the extra generality provided by Eq. (2) is essential is in constructing an estimator for the source function in a medium with non-isotropic scattering.

\subsection{Emergent radiation}

The emergent $\mathrm{rf}$ spectrum for a MC simulation can be derived simply by counting escaping $r$-packets into frequency bins, with light travel-time taken into account as in Sect. 4.2. But even in the spherical case, large $\mathcal{N}$ is then required to keep sampling errors small enough to allow a useful comparison with observed spectra. The solution is to extract the source function from the simulation and then calculate the emergent flux from the formal integral (Lucy 1999b). In a particular $1-\mathrm{D}$ case, the errors in the resulting spectrum correspond to those of a binned spectrum from a simulation with $\mathcal{N}$ increased by a factor of $\sim 320$. For a 3-D SN, where spectra for multiple lines-of-sight must be computed, the binning option is almost useless, and the already large gain factor with the formal integral will be vastly increased.

\section{Gamma ray transport}

In a previous paper (Paper II) on the NLTE transfer of UVOIR radiation in a $\mathrm{SN}$ envelope, the $\mathcal{E}$-packets were all created at the lower boundary, thus implicitly representing the outward diffusion of the energy released by radioactive decays in the deeper interior. But with the computational domain now being the entire ejecta, the creation and transport of $\gamma$-rays must be treated explicitly. In this section, therefore, the concept of indivisible $\mathcal{E}$-packets is extended to cover this aspect of the general spectral synthesis problem for $\mathrm{SNe}$.

In a very recent paper, Milne et al. (2004) have compared results with various $\gamma$-ray transport codes and reviewed the physical processes that must be treated. Their emphasis is on the prediction of $\gamma$-ray spectra rather on the powering of optical emission.

\subsection{Gamma ray lines}

The radioactive decays ${ }^{56} \mathrm{Ni} \rightarrow{ }^{56} \mathrm{Co}$ and ${ }^{56} \mathrm{Co} \rightarrow{ }^{56} \mathrm{Fe}$ each occur with the emission of a spectrum of $\gamma$-ray lines - see Table 1 in Ambwani \& Sutherland (1988). Gamma-rays of 
energy $E_{l}$ are emitted with probability $f_{l}$ when a parent nucleus decays. The total energy emitted per decay is therefore $\sum E_{l} f_{l}$, giving $E_{\mathrm{Ni}}=1.728$ and $E_{\mathrm{Co}}=3.566 \mathrm{MeV}$ for the ${ }^{56} \mathrm{Ni}$ and ${ }^{56} \mathrm{Co}$ nuclei, respectively. The $e$-folding times for these decays are $t_{\mathrm{Ni}}=8.80$ and $t_{\mathrm{Co}}=113.7$ days.

The total $\gamma$-ray energy emitted by the decay sequence ${ }^{56} \mathrm{Ni} \rightarrow{ }^{56} \mathrm{Co} \rightarrow{ }^{56} \mathrm{Fe}$ in the limit $t \rightarrow \infty$ is

$E_{\mathrm{tot}}=\left(E_{\mathrm{Ni}}+E_{\mathrm{Co}}\right) \mathcal{M}_{\mathrm{rad}} / m_{\mathrm{Ni}}$

where $\mathcal{M}_{\mathrm{rad}}$ is the initial mass of ${ }^{56} \mathrm{Ni}$, and $m_{\mathrm{Ni}}$ is the mass of the ${ }^{56} \mathrm{Ni}$ nucleus.

\subsection{Radioactive pellets}

The initial mass of radioactive matter $\mathcal{M}_{\text {rad }}$ is quantized into $\mathcal{N}$ pellets, where $\mathcal{N}=E_{\text {tot }} / \epsilon_{0}$. These pellets have the following property: they emit a single $\gamma$-packet with cmf energy $\epsilon_{0}$ and containing $\gamma$-rays whose cmf photon energy $E_{\gamma}^{\prime}$ corresponds to one of the lines emitted in the decay sequence ${ }^{56} \mathrm{Ni} \rightarrow{ }^{56} \mathrm{Co} \rightarrow$ ${ }^{56} \mathrm{Fe}$. Following this event, a pellet is permanently inert.

As $\mathcal{N} \rightarrow \infty$, this model must yield the correct timedependent $\gamma$-ray line emissivities. To achieve this, we first identify two kinds of pellet: a fraction $E_{\mathrm{Ni}} /\left(E_{\mathrm{Ni}}+E_{\mathrm{Co}}\right)$ are Ni pellets that collectively emit the ${ }^{56} \mathrm{Ni}$ spectrum, while the remainder are Co pellets, and they account for the ${ }^{56}$ Co spectrum.

If a pellet is designated as a $\mathrm{Ni}$ pellet, its decay time is randomly chosen as $t_{\gamma}=-t_{\mathrm{Ni}} \ell n z$; and the emitted $\gamma$-packet is assigned to line $l$ from the ${ }^{56} \mathrm{Ni}$ spectrum with probability $E_{l} f_{l} / \sum E_{l} f_{l}$. On the other hand, a Co pellet's decay time is $t_{\gamma}=$ $-t_{\mathrm{Ni}} \ell n z_{1}-t_{\mathrm{Co}} \ell n z_{2}$, where $z_{1}$ and $z_{2}$ are independent random numbers from $(0,1)$. Two terms are required for the Co pellets since each ${ }^{56} \mathrm{Co}$ nucleus is created in the decay of ${ }^{56} \mathrm{Ni}$ nucleus. Having thus selected $t_{\gamma}$ for a Co pellet, we select a line from the ${ }^{56} \mathrm{Co}$ spectrum as with ${ }^{56} \mathrm{Ni}$.

When a pellet decays, the emitted $\gamma$-packet is assigned a cmf direction vector $\boldsymbol{\mu}^{\prime}$ in accordance with isotropic emission. Thus $\boldsymbol{\mu}^{\prime}=(\sin \theta \cos \phi, \sin \theta \sin \phi, \cos \theta)$, with $\cos \theta=1-2 z$ and $\phi=2 \pi z$. The corresponding rf vector $\mu$ is obtained with the exact aberration formula - e.g., Castor (1972, Eq. (5)). The $\gamma$-packet's initial rf energy is then $\epsilon_{E}=\epsilon_{0} /(1-\mu . v / c)$, where the local $\mathrm{rf}$ velocity $\boldsymbol{v}=\boldsymbol{r} / \mathrm{t}$.

The positions of the pellets at $t_{1}$ are obtained by sampling the distribution of ${ }^{56} \mathrm{Ni}$ predicted by the explosion model. Their positions when they decay are then given by the assumption of homologous expansion.

The $\gamma$-packets emitted in the interval $\left(t_{n}, t_{n+1}\right)$ are added to the $\gamma$ - and $r$-packets still propagating in the ejecta at time $t_{n}$ to form a list of active $\mathcal{E}$-packets whose trajectories are up-dated during this time step.

\subsection{Transport of $\gamma$-packets}

In propagating through the ejecta, $\gamma$-rays create cascades of non-thermal electrons in multiple Compton scatterings off free and bound electrons as well as being absorbed in photoionizations. This redistribution of the energy of the emitted $\gamma$-ray into numerous channels over a substantial volume can nevertheless be modelled with indivisible $\gamma$-packets in such a way that the correct physics emerges as $\mathcal{N} \rightarrow \infty$.

\subsubsection{Events}

As a $\gamma$-packet propagates, it undergoes events, both numerical and physical. The numerical events are: escaping from the grid, reaching the surface of a cell, or coming to the end of the current time step at $t=t_{n+1}$. The currently-included physical events are Compton scattering and photoelectric absorption.

In describing how a $\gamma$-packet's trajectory is computed, it suffices to explain how to find the next event along the trajectory of one packet (cf. Paper II, Sect. 5).

Given the rf position $\boldsymbol{r}$ and direction vector $\boldsymbol{\mu}$ following an event, the next event is identified by computing the distances along the trajectory to all possible events and then selecting the event reached first. Since calculating distances to the numerical events is trivial, we here treat only the physical events.

If $E$ is the $\mathrm{rf}$ energy of photons in a $\gamma$-packet, the $\mathrm{cmf}$ energy is $E^{\prime}=E(1-\mu \cdot v / c)$. Accordingly, in the cmf the $\gamma$-packet sees Compton scattering coefficient $\sigma^{\prime}\left(E^{\prime}\right)$ and absorption coefficient $k^{\prime}\left(E^{\prime}\right)$. But in the rf these transform to $\sigma_{E}=\sigma^{\prime}\left(E^{\prime}\right)(1-\mu . v / c)$ and $k_{E}=k^{\prime}\left(E^{\prime}\right)(1-\mu . v / c)$. Thus, when the radiative transport is carried out in the rf, the absorption, scattering (and emission) coefficients are direction-dependente.g., Castor (1972, Eqs. (2) and (3)).

With these rf coefficients determined, we select the distance $\delta s$ along the trajectory at which a physical event will occur from the standard MC formula

$\left(k_{E}+\sigma_{E}\right) \rho \delta s=-\ell n z$

and this event happens if $\delta s$ is smaller than the distances to the numerical events. Morever, when a physical event happens, it is a Compton scattering if $z<\sigma_{E} /\left(k_{E}+\sigma_{E}\right)$ and a photoelectic absorption if not.

If $\delta s$ is the distance to the selected event, the updated space-time rf coordinates are $\boldsymbol{r}+\delta s \boldsymbol{\mu}$ and $t+\delta s / c$.

\subsubsection{Actions}

Starting with numerical events, we now describe subsequent actions.

If the $\gamma$-packet exits the grid, it escapes to $\infty$, and attention then turns to the next $\gamma$-packet in the list of those active during the current time step. But if the $\gamma$-packet exits only the current cell and not the grid, then it enters the neighbouring cell and the search for the next event proceeds as above. Finally, if the event is the end of the time step at $t_{n+1}$, computation of the trajectory is suspended, and the $\gamma$-packet's current rf data string $\boldsymbol{r}, t, \boldsymbol{\mu}$, $\epsilon_{E}, E$ is stored to await the next time step.

With regard to physical events, the following actions are taken: in the event of a photoelectric absorption, the $\mathcal{E}$-packet's trajectory as a $\gamma$-packet terminates. It now becomes a $k$ - or $i$-packet (cf. Sect. 2.7) with the same cmf energy $\epsilon_{E}^{\prime}$ as the absorbed $\gamma$-packet. Then, since energy storage in the gas is neglected (Sect. 2.4), this $k$ - or $i$-packet converts immediately to an $r$-packet. In a general code, the frequency $v$ of photons in 
this $r$-packet are determined by continuum emission ( $\mathrm{f}$-b or $\mathrm{f}$ - $\mathrm{f}$ ) or line emission following collisional excitation, and these are treatable with the macro-atom formalism (Papers I and II). But here, with grey transport for UVOIR radiation, the emitted $r$-packet can be regarded as bolometric. Its cmf energy is $\epsilon^{\prime}=\epsilon_{E}^{\prime}$ and its cmf direction vector $\mu^{\prime}$ is selected according to isotropic emission. The rf direction vector $\boldsymbol{\mu}$ then follows from the aberration formula, and the rf energy is $\epsilon=\epsilon^{\prime} /(1-\mu . v / c)$. These quantities together with $\boldsymbol{r}$ and $t$ comprise the data string required to initiate the $r$-packet's trajectory in the 3-D code for UVOIR radiation (Sect. 4).

In the event of a Compton scattering, we first find the scattering angle $\Theta$ by randomly selecting $\cos \Theta$ from a look-up table of the percentiles of the $\cos \Theta$ probability distribution as functions of the incident $\gamma$-ray energy. A $\gamma$-ray scattered through angle $\Theta$ has its incident energy $E^{\prime}=\tilde{E}^{\prime} m_{\mathrm{e}} c^{2}$ reduced to $f_{\mathrm{C}} E^{\prime}$, where $f_{\mathrm{C}}=1 /\left[1+\tilde{E}^{\prime}(1-\cos \Theta)\right]$, with the remaining energy transferred to a Compton electon. Thus the energy is divided in the ratio $f_{\mathrm{C}}: 1-f_{\mathrm{C}}$. Accordingly, since $\mathcal{E}$-packets are indivisible, the $\gamma$-packet continues as a $\gamma$-packet if $z<f_{\mathrm{C}}$. If not, it becomes an $\mathrm{e}^{-}$packet.

If the packet continues as a $\gamma$-packet, its cmf energy remains $\epsilon_{E}^{\prime}$ but it now contains $\gamma$-rays of cmf energy $f_{\mathrm{C}} E^{\prime}$. To derive the rf values of these quantities, we must select a new direction vector. In the cmf, the incident $\boldsymbol{\mu}_{1}^{\prime}$ and emergent $\boldsymbol{\mu}^{\prime}$ direction vectors are such that $\boldsymbol{\mu}_{1}^{\prime} \cdot \boldsymbol{\mu}^{\prime}=\cos \Theta$. The combination of this with azimuthal angle $\Phi=2 \pi z$, where azimuth is defined with respect to polar direction $\boldsymbol{\mu}_{1}^{\prime}$, determines $\boldsymbol{\mu}^{\prime}$. The aberration formula then gives $\boldsymbol{\mu}$ and so, in the rf, the emergent $\gamma$-packet has energy $\epsilon_{E}^{\prime} /(1-\boldsymbol{\mu} . \boldsymbol{v} / c)$ and contains $\gamma$-rays with photon energies $f_{\mathrm{C}} E^{\prime} /(1-\boldsymbol{\mu} \cdot \boldsymbol{v} / c)$. The next event for this $\gamma$-packet is now searched for as described in Sect. 3.3.1.

On the other hand, if the $\gamma$-packet converts to an $\mathrm{e}^{-}$packet, we assume in situ degradation into a bolometric $r$-packet with cmf energy $\epsilon^{\prime}=\epsilon_{E}^{\prime}$. The rf data string needed to initiate its subsequent trajectory is now computed as for an $r$-packet created by photoelectric absorption - see above.

\subsection{Test}

The above treatment is a straightforward extension of the indivisible $\mathcal{E}$-packet idea to $\gamma$-ray transport. Nevertheless, it should still be tested against the traditional MC treatment in which the MC quanta are photons (e.g. Colgate et al. 1980; Ambwani \& Sutherland 1988). Such a test has been carried out by computing $\gamma$-ray deposition in a spherical SN. For simplicity, effects of $O(v / c)$ are neglected and photoelectric absorption acts only as a guillotine when $E$ drops below $100 \mathrm{keV}$.

In one calculation, the pellets emit a single $\gamma$-packet as described above. In the comparison calculation, each pellet emits multiple photon packets with photon energies $E_{l}$ and weights $\propto E_{l} f_{l}$, thus representing the appropriate $\gamma$-ray line spectrum. Each such packet then undergoes several Compton scatterings, at each of which the energy of the Compton electron is deposited in situ. This continues until a photoelectric absorption deposits the remaining energy. The two deposition profiles converge to one another as $\mathcal{N} \rightarrow \infty$.

\subsection{Energy deposition rate}

In the present code, an explicit determination of $\mathcal{H}_{\gamma}^{\prime}$, the heating rate due to the deposition of $\gamma$-ray energy, is not required. The assumption of local thermal equilibrium implies that absorbed $\gamma$-packets are immediately re-emitted as $r$-packets (Sect. 3.3.2) and their subsequent propagation is through matter with a temperature-independent grey absorption coefficient. But in a more general code, the thermal history of the ejecta must be calculated, and this requires $\mathcal{H}_{\gamma}^{\prime}$. In any case, this quantity is needed here for the solution with moment equations (Sect. 5).

In Sect. 3.3, two deposition processes are considered. First there is the loss of energy to Compton electrons, which occurs at the rate

$\mathcal{L}_{C}^{\prime}=4 \pi \int \bar{f}\left(E^{\prime}\right) \sigma^{\prime}\left(E^{\prime}\right) J_{E^{\prime}}^{\prime} \mathrm{d} E^{\prime}$

where $\bar{f}\left(E^{\prime}\right)$ is the expected fraction of $E^{\prime}$ transferred to the Compton electron and $J_{E^{\prime}}^{\prime}$ is the cmf mean intensity at photon energy $E^{\prime}$. Second, there is the loss in photoionizations, which occurs at the rate

$\mathcal{L}_{I}^{\prime}=4 \pi \int k^{\prime}\left(E^{\prime}\right) J_{E^{\prime}}^{\prime} \mathrm{d} E^{\prime}$.

Estimators for these rates can be derived by applying Eq. (2) in the cmf. But note that the transport of packets is carried out in the rf. If $\delta s$ is the distance between consecutive events for a $\gamma$-packet propagating in the $\mathrm{rf}$, then, to $O(v / c)$, in the $\mathrm{cmf}$, $\delta s^{\prime}=\delta s(1-\mu . v / c)$. Accordingly, since we also have $\epsilon_{E}^{\prime}=$ $\epsilon_{E}(1-\mu . v / c)$, the reqired estimators are

$\mathcal{L}_{C}^{\prime}=\frac{\epsilon_{0}}{\Delta t} \frac{1}{V} \sum \bar{f}\left(E^{\prime}\right) \sigma^{\prime}\left(E^{\prime}\right) \frac{\epsilon_{E}}{\epsilon_{0}} \delta s(1-2 \mu . v / c)$

and

$\mathcal{L}_{I}^{\prime}=\frac{\epsilon_{0}}{\Delta t} \frac{1}{V} \sum k^{\prime}\left(E^{\prime}\right) \frac{\epsilon_{E}}{\epsilon_{0}} \delta s(1-2 \mu . v / c)$.

These summations are over all trajectory segments $\delta s$ in $V$. Thus there is no restriction to packets that undergo Compton scatterings (Eq. (7)) or photoelectric absorptions (Eq. (8)) in $V$. Accordingly, the resulting deposition rate $\mathcal{H}_{\gamma}^{\prime}=\mathcal{L}_{\mathrm{C}}^{\prime}+\mathcal{L}_{\mathrm{I}}^{\prime}$ is more accurate than the crude estimate obtained by summing the cmf energies of $\gamma$-packets that terminate their trajectories in $V$.

\subsection{Gamma-ray spectra}

A by-product of this 3-D time-dependent treatment of $\gamma$-ray transport is the prediction of $\gamma$-ray spectra, observations of which have potential for detecting asymmetries in SN explosions (Höflich 2002; Hungerford et al. 2003). Crude spectra can be obtained simply by binning escaping $\gamma$-packets according to their rf energies $E$. But for high quality results, especially for the orientation-dependent spectra of an asymmetric $\mathrm{SN}$, the formal integral should be used (Sect. 2.10).

\subsection{Future improvements}

Additional physical processes can be readily incorporated into this $\mathcal{E}$-packet treatment of $\gamma$-ray transport. 


\subsubsection{Positron transport}

Following Ambwani \& Sutherland (1988, Table 1), positrons emitted in ${ }^{56} \mathrm{Co}$ are here assumed to annihilate in situ. But at late times, the transport of positrons should be followed until they escape or deposit their rest and kinetic energies in the ejecta. This requires that we allow the Co pellets to emit $\mathrm{e}^{+}$packets instead of the $\gamma$-packets with $E=0.511 \mathrm{MeV}$.

\subsubsection{Transport of non-thermal electrons}

In treating Compton scattering, we assume that Compton electrons degrade in situ. The basis for this is the short stopping distance of $\mathrm{MeV}$ electrons compared to $\mathrm{MeV} \gamma$-rays (Colgate et al. 1980). Nevertheless, we may eventually wish to follow the motion of the $\mathrm{e}^{-}$-packets, especially at late epochs.

\subsubsection{Pair production}

In addition to Compton scattering and photoelectric absorption, a $\gamma$-ray with $E>2 m_{\mathrm{e}} c^{2}$ can tranform to an $\mathrm{e}^{+}-\mathrm{e}^{-}$pair as it passes an atomic nucleus - see, e.g., Ambwani \& Sutherland (1988) - with each of the pair having kinetic energy (ke) $E / 2-m_{\mathrm{e}} c^{2}$. In stopping, the positron releases energy $E / 2+m_{\mathrm{e}} c^{2}$, comprising its ke plus the rest energy $2 m_{\mathrm{e}} c^{2}$ radiated when the positron annihilates with an ambient electron. In contrast, stopping the electron releases only its ke.

Accordingly, when treated with indivisible $\mathcal{E}$-packets, pair production converts a $\gamma$-packet into an $e^{+}$-packet with probability $1 / 2+q$ or into an $\mathrm{e}^{-}$-packet with probability $1 / 2-q$, where $q=m_{\mathrm{e}} c^{2} / E^{\prime}$. In either case, the cmf energy of the packet is that of the incident $\gamma$-packet $\epsilon_{E}^{\prime}$. If in situ deposition is not assumed, the selected packet's motion is then followed to escape or deposition. Note that a return to the pair production event to follow the other member of the pair is not required (cf. Paper I, Sect. 1).

\subsubsection{Rare radioactivites}

If the evolution of the ejecta is followed to late epochs ( $>1000$ days), then rare but long-lived radioactive isotopes such as ${ }^{57} \mathrm{Co},{ }^{44} \mathrm{Ti}$ and ${ }^{22} \mathrm{Na}$ must be included (e.g., Woosley et al. 1989). This just requires additional types of pellet.

\section{Transport of UVOIR radiation}

In a previous paper (Paper II) the NLTE transfer of UVOIR radiation in a $\mathrm{SN}$ envelope of pure $\mathrm{H}$ was treated using and extending the macro-atom formalism (Paper I). Here, given the emphasis on time dependence and 3-D, we simplify to a grey absorption coefficient.

\subsection{Transport of $r$-packets}

As with $\gamma$-packets, the transport of $r$-packets is carried out in a sequence of MC simulations for the time steps $t_{n} \rightarrow t_{n+1}$. Each of these simulations directly follows the corresponding one for $\gamma$-packets (Sect. 3.3).

\subsubsection{Sources of $r$-packets}

The $r$-packets that propagate in the ejecta in the time interval $\left(t_{n}, t_{n+1}\right)$ have several sources. First are those that did not escape during the previous time step. For these, we have the rf data string $(\boldsymbol{r}, t, \boldsymbol{\mu}, \epsilon)$, and so their trajectories can be continued from time $t_{n}$. Second are those created during the current time step as $\gamma$-packets are eliminated. This occurs either by photoelectric absorption or by the stopping of Compton electrons (Sect. 3.3.2). In either case, the $\gamma$ transport routine provides the rf data string $(\boldsymbol{r}, t, \boldsymbol{\mu}, \boldsymbol{\epsilon})$ needed to initiate their trajectories as $r$-packets.

A third source is specific to the first time step. Radiant energy emitted by pellets that decay at times prior to $t_{1}$ must be accounted for. An approximate treatment is as follows: A $\gamma$-packet emitted at $t_{\gamma}<t_{1}$ and position $\boldsymbol{r}_{\gamma}$ is assumed to have converted to an $r$-packet by time $t_{1}$ but to have diffused negligibly relative to matter (position coupling) in the time interval $\left(t_{\gamma}, t_{1}\right)$ so that at $t_{1}$ its rf position is $\boldsymbol{r}_{\gamma}\left(t_{1} / t_{\gamma}\right)$. This is justified by the short mean free paths of photons in these early, high-density phases. The rf direction vector at $t_{1}$ is computed on the assumption of isotropic emission in the $\mathrm{cmf}$.

The energies of these packets must also be specified. Although position coupled, they still do work on the expanding ejecta. An $r$-packet's energy at $t_{1}$ is therefore $\epsilon=\epsilon_{0} t_{\gamma} / t_{1}$.

An additional source of $r$-packets at $t_{1}$ is the radiation generated by shock heating during the explosion. The explosion model's prediction of this radiation can be discretized into $r$-packets of energy $\epsilon_{0}$, the same quantum of energy as for the radioactive pellets (Sect. 3.2). However, such $r$-packets are neglected in this test code.

\subsubsection{Events}

As an $r$-packet propagates, it undergoes numerical and physical events. The numerical events are identical to those for $\gamma$-packets (Sect. 3.3.1). The physical events are absorptions.

In describing how a $r$-packet's trajectory is computed, it suffices to explain how to find the next event along the trajectory of one packet. Given the rf data string $(\boldsymbol{r}, t, \boldsymbol{\mu}, \epsilon)$, the next event is identified by computing the distances along the trajectory to all possible events and then selecting the event reached first. As with $\gamma$-packets, we treat only physical events.

Because the transport of $r$-packets is also carried out in the $\mathrm{rf}$, the anisotropy of the rf absorption coefficient must again be allowed for, as it was for $\gamma$-packets in Sect. 3.3.1. If the grey absorption coefficient per unit volume in the $\mathrm{cmf}$ is $k^{\prime}$, the effective value in the $\mathrm{rf}$ is $k=k^{\prime}(1-\mu . v / c)$, and so the distance $\delta s$ to the absorption event is given by $k \delta s=-\ell n z$. This event happens if this is smaller than the distance to any numerical event. With $\delta s$ thus determined, the coordinates $(\boldsymbol{r}, t)$ are updated as for $\gamma$-packets (Sect. 3.3.1).

\subsubsection{Actions}

For numerical events, the subsequent actions correspond to those for $\gamma$-packets (Sect. 3.3.2). 
If the event is an absorption, we assume instantaneous isotropic re-emission in the cmf. Accordingly, the new rf direction vector $\boldsymbol{\mu}$ is calculated as it was for emitted $\gamma$-packets in Sect. 3.2.

The energy content of the bolometric $r$-packet must also be updated. If the incident packet has rf energy $\epsilon_{1}$ and direction vector $\boldsymbol{\mu}_{1}$, its cmf energy is $\epsilon^{\prime}=\epsilon_{1}\left(1-\mu_{1} \cdot v / c\right)$. Since this is conserved by the absorption-emission event, the updated rf energy is $\epsilon=\epsilon^{\prime} /(1-\mu . v / c)$. Calculation of the post-event data string $(\boldsymbol{r}, t, \boldsymbol{\mu}, \epsilon)$ is now complete, and the search for the next event can begin.

\subsection{Bolometric light curve}

Consider an $r$-packet with post-event data string $(\boldsymbol{r}, t, \boldsymbol{\mu}, \epsilon)$ that is an escapee from the grid. A distant observer at rest in the rf who detects this packet records its arrival at what he perceives to be a time $\tau=t-\mu . r / c$ after the explosion. Thus the pairs $(\epsilon, \tau)$ detected by one such observer is the data set from which he can construct the bolometric light curve of the 3-D SN as seen from his orientation.

In this paper, the 3-D code is tested by applying it to a spherically-symmetric SN. Accordingly, we use all pairs $(\epsilon, \tau)$ to construct its orientation-averaged bolometric light curve (Sect. 6.3).

\section{Solution with moment equations}

In order to test the 3-D code described in Sects. 3 and 4, we apply it in Sect. 6 to a spherically-symmetric SN. This allows the code to be tested against a solution of the same problem obtained by numerical integration of the time-dependent RTE.

\subsection{Castor's equations}

Castor (1972) has given a general treatment, accurate to $O(v / c)$, of radiative transfer in spherically-symmetric flows. In particular, he derives the zeroth and first frequency-integrated moment equations in the cmf. These two equations, applied to a homologously expanding flow with grey absorption, are the basis of the comparison calculation.

If we again neglect the contributions of the gas to energy balance (Sect. 2.4), the zeroth moment equation is

$\frac{\mathrm{d} U_{\mathrm{R}}^{\prime}}{\mathrm{d} t}+\rho \frac{\partial L^{\prime}}{\partial \mathcal{M}_{r}}+\frac{4 U_{\mathrm{R}}^{\prime}}{t}=\mathcal{H}_{\gamma}^{\prime}$

where the dependent variables are $U_{\mathrm{R}}^{\prime}$, the cmf energy density of radiation, and $L^{\prime}$, the cmf luminosity variable. The independent variables are elapsed time $t$ and the mass coordinate $\mathcal{M}_{r}$. Note that time derivatives in the moment equations are Lagrangian.

The first moment equation is

$\frac{1}{c} \frac{\mathrm{d} L^{\prime}}{\mathrm{d} t}+\alpha_{1} \frac{\partial P_{\mathrm{R}}^{\prime}}{\partial \mathcal{M}_{r}}+\alpha_{2}\left(3 P_{\mathrm{R}}^{\prime}-U_{\mathrm{R}}^{\prime}\right)=-\left(k^{\prime}+\frac{2}{c t}\right) L^{\prime}$

where $\alpha_{1}=16 \pi^{2} r^{4} \rho c, \alpha_{2}=4 \pi r c$, and the third dependent variable $P_{\mathrm{R}}^{\prime}$ is the $\mathrm{cmf}$ radiation pressure.

\subsection{Closure approximation}

To make this system determinate, an approximate formula relating the three moments must be imposed. Here we adopt Eddington's approximation,

$P_{\mathrm{R}}^{\prime}=U_{\mathrm{R}}^{\prime} / 3$

which is used to eliminate $P_{\mathrm{R}}^{\prime}$ from Eq. (10). This leaves two partial differential equations (PDEs) in the two variables $U_{\mathrm{R}}^{\prime}\left(\mathcal{M}_{r}, t\right)$ and $L_{\mathrm{R}}^{\prime}\left(\mathcal{M}_{r}, t\right)$.

\subsection{Boundary conditions}

The PDEs must be solved subject to appropriate boundary conditions. Clearly, at the SN's centre,

$L^{\prime}(0, t)=0$

while, at the surface, Eddington's boundary condition $F(0)=$ $2 J(0)$ gives

$L^{\prime}(\mathcal{M}, t)=2 \pi R_{\max }^{2} c U_{R}^{\prime}(\mathcal{M}, t)$.

Here $R_{\max }(t)=v_{\max } t$, and $\mathcal{M}$ is the total mass of the ejecta.

\subsection{Initial conditions}

In addition, initial conditions are required at $t=t_{1}$. As in the MC code (Sect. 4.1.1), we assume that diffusion of radiation relative to matter is still negligible (Sect. 4.1.1) at $t_{1}$, and so

$L^{\prime}\left(\mathcal{M}_{r}, t_{1}\right)=0$.

An initial condition for $U_{\mathrm{R}}^{\prime}$ is derived as follows: For $t<t_{1}$, negligible relative diffusion of $\gamma$-rays implies in situ deposition, so that $\mathcal{H}_{\gamma}^{\prime}=4 \pi j_{\gamma}^{\prime}$, the integrated $\gamma$-ray emissivity in the cmf. Accordingly, from Eq. (9), the required initial condition is given by integrating the ordinary differential equation (ODE)

$\frac{\mathrm{d} U_{\mathrm{R}}^{\prime}}{\mathrm{d} t}+\frac{4 U_{\mathrm{R}}^{\prime}}{t}=4 \pi j_{\gamma}^{\prime}$

which can be done analytically.

If $f\left(\mathcal{M}_{r}\right)$ is the mass fraction of ${ }^{56} \mathrm{Ni}$ in the mass shell $\mathcal{M}_{r}$ at $t=0$, then

$4 \pi j_{\gamma}^{\prime}=S(t) f \rho / m_{\mathrm{Ni}}$

where $S(t)$, the energy released per ${ }^{56} \mathrm{Ni}$ nucleus, is given by

$S(t)=\frac{E_{\mathrm{Ni}}}{t_{\mathrm{Ni}}} \mathrm{e}^{-t / t_{\mathrm{Ni}}}+\frac{E_{\mathrm{Co}}}{t_{\mathrm{Co}}-t_{\mathrm{Ni}}}\left(\mathrm{e}^{-t / t_{\mathrm{Co}}}-\mathrm{e}^{-t / t_{\mathrm{Ni}}}\right)$.

Since each term in Eq. (17) has the same form and Eq. (15) is linear, we consider a single exponential - i.e., $S(t)=$ $E_{*} / t_{*} \mathrm{e}^{-t / t_{*}}$, and we note also that homologous expansion implies that $\rho \propto 1 / t^{3}$. Integration of Eq. (15) then gives

$U_{\mathrm{R}}^{\prime}=E_{*}\left(\frac{t_{*}}{t}-\left[1+\frac{t_{*}}{t}\right] \mathrm{e}^{-t / t_{*}}\right) f \rho / m_{\mathrm{Ni}}$.

This solution can be applied to each term in Eq. (17) to construct the complete solution. But with $t_{1} \ll t_{\mathrm{Ni}}$, the first $\left({ }^{56} \mathrm{Ni}\right)$ term suffices.

Note that, in deriving Eq. (18), we assumed $U_{\mathrm{R}}^{\prime}\left(\mathcal{M}_{r}, t\right)=0$ at $t=0$. This is consistent with the neglect of shock heating in the MC code (Sect. 4.1.1). 


\subsection{Solution technique}

Equations (9) and (10) are solved in the same way as are the equations of stellar evolution when the $\dot{P}$ and $\dot{T}$ terms are included in the energy equation (e.g., Schwarzschild 1958, p. 100). Thus, the time derivatives of $U_{\mathrm{R}}^{\prime}$ and $L^{\prime}$ are replaced by backward difference formulae. Then, since the solution at earlier time steps is known, the PDE problem is effectively simplified to a two-point boundary value problem for ODEs. When the space derivatives in the ODEs are approximated by difference formulae, the resulting algebraic system can be solved with an elimination procedure (Henyey method). Here, a slight generalization of the procedure described by Richtmyer (1957, pp. 101-104) has been followed.

In stellar evolution codes, it is still current practice (A. Weiss, private communication) to approximate the time derivative of a variable $Q$ at time $t_{n}$ with the formula

$\left(\frac{\mathrm{d} Q}{\mathrm{~d} t}\right)_{n} \approx \frac{\Delta Q_{n-1}}{\Delta t_{n-1}}$

where $\Delta$ denotes the forward difference operator, so that $\Delta t_{n-1}=t_{n}-t_{n-1}$. This formula is not centred and thus has error $O(\Delta t)$. But higher accuracy can be achieved by using more than one previous model - e.g, Richtmyer (1957, p. 94, item 9). If the two previous models at $t_{n-1}$ and $t_{n-2}$ are used, the appropriate difference approximation is obtained by fitting a parobola and then evaluating its derivative at $t_{n}$. The resulting formula is

$\left(\frac{\mathrm{d} Q}{\mathrm{~d} t}\right)_{n} \approx(1+a) \frac{\Delta Q_{n-1}}{\Delta t_{n-1}}-a \frac{\Delta Q_{n-2}}{\Delta t_{n-2}}$

where $a=\Delta t_{n-1} /\left(\Delta t_{n-1}+\Delta t_{n-2}\right)$. Thus, with constant time steps, $a=1 / 2$. The gain in accuracy is investigated in Sect. 6.2.

In starting the integration of the PDEs, the theory of Sect. 5.4 is used to provide the previous solutions required by Eqs. (19) and (20) as well as initial estimates of $U_{\mathrm{R}}^{\prime}$ and $L^{\prime}$ for the first Henyey iteration.

\subsection{Light curve}

The solution of the PDEs yields the two functions $U_{\mathrm{R}}^{\prime}\left(\mathcal{M}_{r}, t\right)$ and $L^{\prime}\left(\mathcal{M}_{r}, t\right)$. Thus we directly get the bolometric light curve $L^{\prime}(t)$ seen by a cmf observer at $\mathcal{M}_{r}=\mathcal{M}$, the surface of the SN. But the quantity of interest is the light curve seen by a distant observer at rest in the rf. This can be calculated using $\mathcal{E}$-packets as follows:

In a small interval $\Delta t$ at $t$, the surface emits $N r$-packets at random times $t_{n}$ in $\Delta t$. These all have $\mathrm{cmf}$ energy $\epsilon=$ $L^{\prime}(t) \Delta t / N$, and their direction cosines are $\mu_{n}^{\prime}=\sqrt{z}$, corresponding to zero limb-darkening, an assumption consistent with Eddington's approximations. Their direction cosines $\mu_{n}$ in the rf are given by the aberration formula, and their $\mathrm{rf}$ energies are then $\epsilon_{n}=\epsilon /\left(1-\mu_{n} v_{\max } / c\right)$. The distant observer perceives the $n$th packet as having been emitted at elapsed time $\tau_{n}=t_{n}-\mu_{n} R_{\max } / c$, and so the $N$ pairs $\left(\epsilon_{n}, \tau_{n}\right)$ represent the contribution of the interval $\Delta t$ to the bolometric light curve seen by this observer. Summing over all intervals $\Delta t$ then gives the complete light curve (cf. Sect. 4.2).

\section{Numerical results}

After first investigating the accuracy of bolometric light curves for spherical SN obtained with the $\mathrm{cmf}$ moment equations, this section uses such a light curve to check the 3-D MC code described in Sects. 3 and 4.

\subsection{Model}

The model SN is closely similar to that used by Pinto \& Eastman (2000) to investigate the parameter sensitivity of type Ia light curves. The ejecta has uniform density and its basic parameters are: $\mathcal{M}=1.39 \mathcal{M}_{\odot}, \mathcal{M}\left({ }^{56} \mathrm{Ni}\right)=0.625 \mathcal{M}_{\odot}$, and $v_{\max }=10^{4} \mathrm{~km} \mathrm{~s}^{-1}$. The ${ }^{56} \mathrm{Ni}$ is assumed to be stongly concentrated in the central core. Thus, $f\left(\mathcal{M}_{r}\right)=1$ for $\mathcal{M}_{r}<0.5 \mathcal{M}_{\odot}$ and then drops linearly to zero at $\mathcal{M}_{r}=0.75 \mathcal{M}_{\odot}$.

The grey absorption coefficient for UVOIR radiation is $k^{\prime} / \rho=0.1 \mathrm{~cm}^{2} \mathrm{~g}^{-1}$, and the photoelectric absorption coefficient $k_{E}^{\prime}$ for $\gamma$-rays is derived from Eq. (3) of Ambwani \& Sutherland (1988) with nuclear charge $Z=14$.

\subsection{Accuracy of moment solution}

Numerical solutions of Castor's equations have been obtained in order to test the MC code against an RTE treatment accurate to $O(v / c)$. But the neglect of higher order terms in $v / c$ is not the only source of error in the RTE solutions. Larger errors may arise due to the closure approximation (Sect. 5.2) and the difference approximation of time derivatives (Sect. 5.5).

The moment equations are solved on a uniformly-spaced grid with 400 grid points, the innermost at $10^{-3} R_{\max }$. The energy deposition rate $\mathcal{H}_{\gamma}^{\prime}$ in Eq. (9) is derived with a 1-D version of the MC code described in Sect. 3 using the estimators given in Eqs. (7) and (8). The number of radioactive pellets is $\mathcal{N}=10^{7}$.

To investigate sensitivity to the approximation of time derivatives, two sequences of light curves are computed, one using Eq. (19) to evaluate $\mathrm{d} U_{\mathrm{R}}^{\prime} / \mathrm{d} t$ and $\mathrm{d} L_{\mathrm{R}}^{\prime} / \mathrm{d} t$, the other using Eq. (20). Along each sequence, $\Delta \log t$ varies, thereby determining the rate of convergence as $\Delta \log t \rightarrow 0$. For each light curve, $M_{\mathrm{bol}}^{\max }$, the peak rf bolometric magnitude, is obtained by parabolic fitting.

The values of $M_{\mathrm{bol}}^{\max }$ as a function of $\Delta \log t$ are plotted in Fig. 1, together with the value $M_{\mathrm{bol}}^{\max }=-19.181 \mathrm{ob}-$ tained by extrapolating to infinitesimal time step. Error bands of \pm 0.05 mag. are also drawn.

Figure 1 shows, as expected, that errors grow linearly with Eq. (19) and quadratically with Eq. (20). From this plot, we find that accuracy $<0.05 \mathrm{mag}$. requires $\Delta \log t<0.048$ with Eq. (20) but $\Delta \log t<0.009$ with Eq. (19). Clearly, for high precision, Eq. (20) is preferred and is indeed used in Sect. 6.3.

Although this test refers to the cmf moment equations, it is surely relevant generally to numerical solutions of the time-dependent RTE. Accurate difference approximations for time derivatives are necessary to limit the accumulation of errors as one integrates forward in time. And of course the same remark applies to stellar evolution codes. 


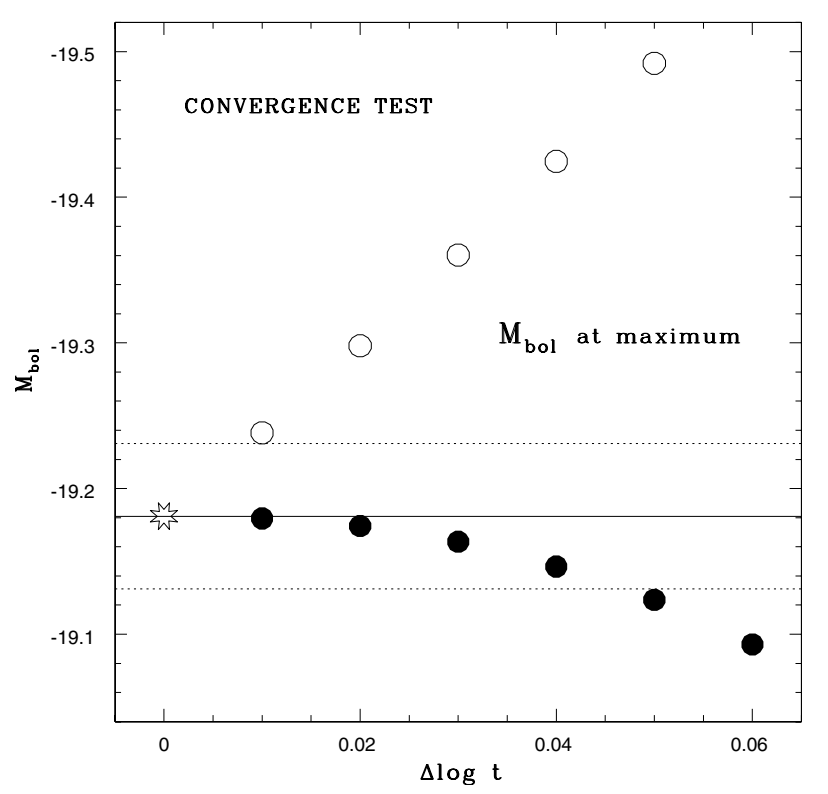

Fig. 1. Bolometric magnitude at maximum as function of time step $\Delta \log t$. Time derivatives are approximated with Eq. (19) (open circles) or Eq. (20) (filled circles). The extrapolation to vanishingly small time step is shown (star) as are \pm 0.05 mag error bands.

\subsection{Bolometric light curves}

In Fig. 2, the rf bolometric light curve computed with the 3-D MC code is compared with that derived from the cmf moment equations. The MC light curve is from a simulation in which the initial distribution of radioactive matter is represented by $\mathcal{N}=4 \times 10^{6}$ pellets, with the resulting $\gamma$ - and $r$-packets propagating in a $100^{3}$ grid. The calculation starts at $\log t_{1}$ (dys) $=0.3$, and the time steps are $\Delta \log t=0.01$. The rf light curve is derived from escaping $r$-packets as described in Sect. 4.2. Thus, for packets with arrival times $\tau$ in the interval $\left(t_{n}, t_{n+1}\right)$, the values of $\epsilon$ are summed to obtain an estimate of $L_{n+1 / 2}$.

The rf light curve derived from the PDEs of Sect. 5.1 is also plotted in Fig. 2. This is derived from the cmf light curve $L^{\prime}(t)$ as described in Sect. 5.6. As for the calculations of Sect. 6.2, the spherical $\mathrm{SN}$ is modelled with 400 shells, and $\mathcal{N}=10^{7}$ in the 1-D $\gamma$-ray code.

Figure 2 shows that the two bolometric light curves are in good agreement over an extended time interval, from well before to long after maximum light at $t=15.3$ days. This implies a corresponding degree of agreement in the $\gamma$-ray energy deposition rates. Nevertheless, these are also plotted in Fig. 2. For the 3-D code, the deposition rate is calculated simply by summing the energies of $\gamma$-packets as they convert to $r$-packets. But for the 1-D code, the estimators defined by Eqs. (7) and (8) are used to calculate $\mathcal{H}_{\gamma}^{\prime}$, which is then summed over shells.

To investigate the precision of the 3-D light curve in more detail, the residuals relative to the PDE solution are plotted in Fig. 3. This plot reveals large systematic residuals at early times on the rising branch of the light curve but that these decrease with time becoming quite small for $t \gtrsim 8$ dys. For the interval 10-50 days, the mean residual is $-0.008 \mathrm{mag}$. A residual of

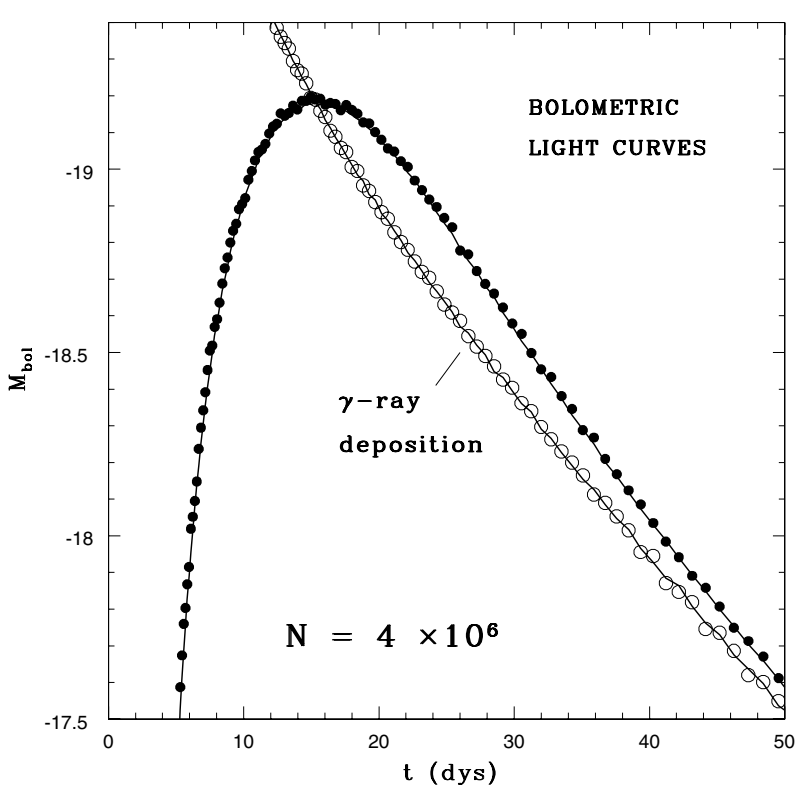

Fig. 2. Comparison of bolometric light curves. The light curve obtained with the 3-D Monte Carlo code (filled circles) compared to that obtained from the moment equations (solid line). The corresponding $\gamma$-ray deposition curves are also plotted.

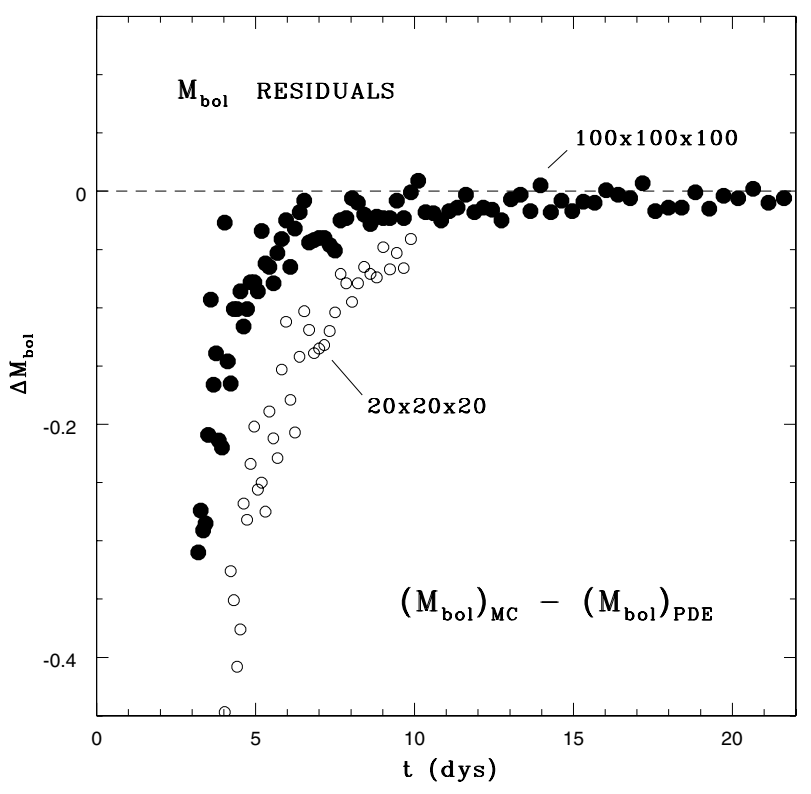

Fig. 3. Light curve residuals. The differences between the bolometric light curves plotted in Fig. 2 as a function of elapsed time $t$. Residuals for 3-D MC calculations with $100^{3}$ (filled circles) and $20^{3}$ (open circles) grids are shown.

this order may reflect errors in the PDE solution since this relies on Eddington's approximations.

The large residuals on the rising branch have been traced to inadequate spatial resolution of the distribution of ${ }^{56} \mathrm{Ni}$ in the 3 -D code, despite the $100^{3}$ grid. Because ${ }^{56} \mathrm{Ni}$ is uniformly distributed within each cubical cell, the function $f\left(\mathcal{M}_{r}\right)$ (Sect. 6.1) is slightly broadened relative to its more accurate representation in the 1-D calculation. This results in some released radioactive energy reaching the surface slightly early, giving a brighter MC light curve. This explanation is confirmed by 
finding that these residuals become larger with a coarser grid. To illustrate this, Fig. 3 includes the residuals for $t<10$ days with a $20^{3}$ grid.

This comparison with the PDE solution shows that, with a fine enough grid, the MC code described in Sects. 3 and 4 is capable of carrying out high precision transport calculations accurate to $O(v / c)$ for $\gamma$-rays and UVOIR radiation propagating in a $3-\mathrm{D} \mathrm{SN}$.

\subsection{Diagnostics}

Among the merits of using $\mathcal{E}$-packets is conservation of energy to high precision and the ready monitoring of energy transformations within the configuration.

\subsubsection{Energy conservation}

Energy conservation for the ejecta implies that

$E_{\infty}(t)+E_{R}(t)-W(t)=E_{\gamma}(t)$.

Here $E_{\gamma}(t)$ is the total energy released by radioactive decays in the time interval $(0, t), E_{\infty}(t)$ is the total energy lost through the surface in $(0, t)$, and $E_{R}(t)$ is the radiant energy stored in the ejecta at time $t$, all these quantities being evaluated in the rf. Finally, $W(t)$ is the total work done in $(0, t)$ by radiation interacting with the expanding ejecta.

Estimators for the quantities in Eq. (21) are sums over packets. Thus, $E_{\gamma}(t)$ is the sum of the initial rf energies $\epsilon_{E}$ of $\gamma$-packets emitted by radioactive pellets (Sect. 3.2); $E_{\infty}(t)$ is the sum of the rf energies $\epsilon_{E}$ and $\epsilon$ of $\gamma$ - and $r$-packets that escape the grid in $(0, t)$; and $E_{\mathrm{R}}(t)$ is the sum of the rf energies of packets still propagating within the ejecta at time $t$. Finally, $W(t)$ is the sum over all physical events in $(0, t)$ of $\Delta \epsilon=\epsilon_{1}-\epsilon_{2}$, where $\epsilon_{1}$ and $\epsilon_{2}$ are an $\mathcal{E}$-packet's incident and emergent rf energies, respectively.

At all time steps in the calculation of Sect. 6.3, the left- and right-hand sides of Eq. (21) agree to better than 1 part in $10^{12}$.

\subsubsection{Energy flows}

The time variations of the quantities in Eq. (21) are plotted in Fig. 4, where the unit of energy is $E_{\text {tot }}$ from Eq. (3). For the adopted parameters, $E_{\mathrm{tot}}=1.14 \times 10^{50} \mathrm{erg}$.

Figure 4 illustrates the strong departures from stationarity. In the early dense phases ( $t \lesssim 5$ days), energy released by radioactive decays is trapped within the ejecta, resulting in increasing $E_{\mathrm{R}}(t)$ while $E_{\infty}(t)$ remains close to zero. This continues until $E_{\mathrm{R}}(t)$ reaches a maximum of 0.085 at $t=9.6$ days. Thereafter, the dropping density allows the trapped radiation to be released, resulting in a sharp increase in $E_{\infty}(t)$ and concomitant decrease in $E_{\mathrm{R}}(t)$. For $t \gtrsim 35$ days, energy storage is inconsequential ( $E_{\mathrm{R}} \lesssim 0.02$ ); stationarity then becomes a good approximation, as is evident from the closely similar slopes of $E_{\gamma}(t)$ and $E_{\infty}(t)$.

The maximum of $E_{\mathrm{R}}(t)$ is a rough measure of the highest fraction of $\mathcal{E}$-packets active at one time and for which, therefore, storage space must be allocated. In fact, because many of

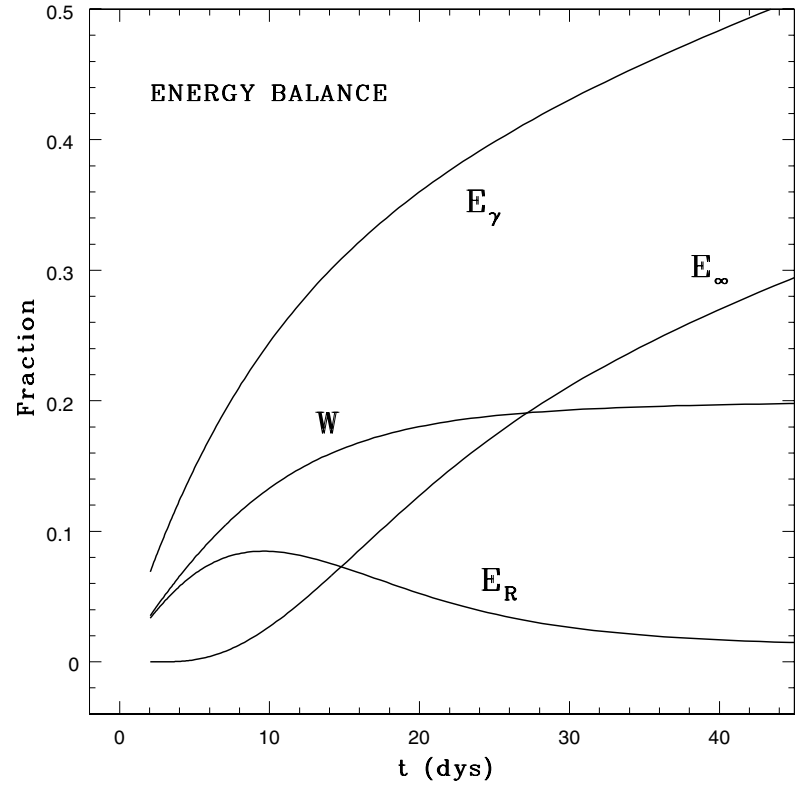

Fig. 4. Energy fractions as functions of elapsed time $t$. Quantities plotted are $E_{\gamma}$, the integrated energy released by radioactive decays, $E_{\infty}$, the integrated energy emitted to $\infty, W$, the work done on the expanding ejecta, and $E_{\mathrm{R}}$, the radiant energy stored in the ejecta. The unit of energy is $E_{\text {tot }}-$ see Eq. (3) - so that $E_{\gamma}(\infty)=1$.

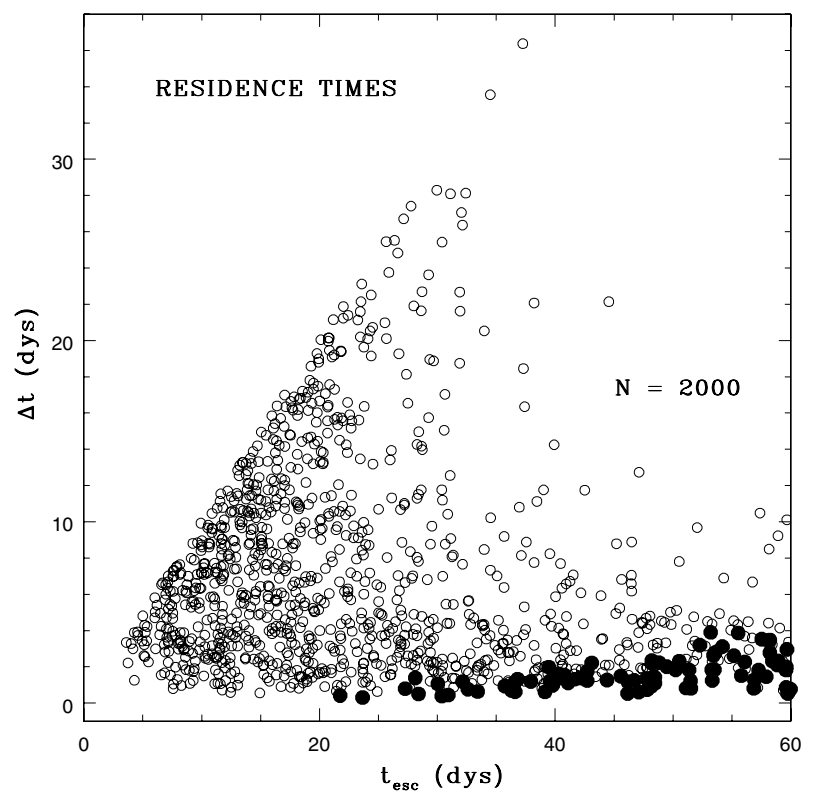

Fig. 5. Residence times $\Delta t=t_{\mathrm{esc}}-t_{\gamma}$ plotted against $t_{\mathrm{esc}}$. Escaping $r$ packets (open circles) and $\gamma$-packets (filled circles) are indicated.

the packets trapped at $t=9.6$ days have lost energy by doing work on the expanding ejecta, the active fraction is larger. In the calculation reported here, the peak active number is $7.2 \times$ $10^{5}$ at $t=9.2$ days - i.e., $0.18 \mathrm{~N}$.

Non-stationarity is also illustrated by Fig. 5, which plots residence against escape times for $\mathcal{E}$-packets in a small simulation with $\mathcal{N}=2000$. For $t \lesssim 20$ days, we see that it is not uncommon for escaping $r$-packets to have residence times close to the age of the SN. Thereafter, the lowered density allows packets to escape readily and typical residence times 
drop to $\sim$ a few days. Note also that no $\gamma$-packets escape until $t \gtrsim 20$ days.

In stationary problems, or this problem with $c=\infty$, a MC simulation must continue until all packets have escaped. This limits the usefulness of MC methods for optically thick media. But here a packet initially at large optical depth escapes when that depth has dropped to $\sim 1$ because of expansion. Figure 5 illustrates this effect.

For this problem, the difficulty with an unacceptably large number of events before escape would reappear if $t_{1} \rightarrow 0$ were necessary for accuracy. Fortunately, the position coupling assumption (Sect. 4.1.1) is well justified for $t \lesssim 1$ day.

\section{Conclusion}

The aim of this paper has been to initiate a MC approach to computing light curves and spectra for 3-D SN explosions. The adopted procedure is based on indestructible and indivisible $\mathcal{E}$-packets as the MC quanta. Although not called upon for this test problem, the attraction of this approach in the long-term for this and other multi-dimensional NLTE problems is that it allows the constraints of thermal and statistical equilibrium to be incorporated into the scheme via the concept of macro-atoms. Moreover, the iterations required to achieve full self-consistency between radiation, level populations and the electron temperature can be carried out with geometry-independent $\Lambda$-iterations.

The calculations reported in Sect. 6 demonstrate that high photometric precision can be achieved with MC methods for time-dependent transport calculations in 3-D. Contributing strongly to maintaining accuracy are the radioactive pellets introduced in Sect. 3.2 since they result in a seamless transition from energy transport by $\gamma$-rays to that by UVOIR radiation. This avoids the loss of accuracy that might arise if the $\gamma$-ray deposition profile were separately calculated and then randomly sampled to create UVOIR radiation. An incidental benefit is simplified coding.

In Sect. 1, this investigation was described as providing a platform onto which more detailed treatments of matter-radiation interactions can be added. Thus the already detailed modelling of $\gamma$-ray transport can be further improved as discussed in Sect. 3.7. Also $\mathcal{L}_{\mathrm{C}}^{\prime}$, the rate of energy deposition in the form of Compton electrons, is available from Eq. (7) as the source term for a Spencer-Fano calculation of the rates at which non-thermal electrons lose energy to thermal electrons and in ionizing and exciting atoms. Finally, the NLTE transfer of UVOIR radiation can be implemented following the successful test for a pure $\mathrm{H}$ envelope in Paper II.

An encouraging aspect of this agenda is that the physics missing from the present code is well understood, even though many relevant cross sections are still poorly known. But a discouraging aspect is that the demands on computer power are such that useful results for a physically- realistic 3-D model are not feasible at present with a single workstation. Nevertheless, as argued in Paper II, an attractive option is to implement these methods on a computer with numerous parallel processors. But even then, it is probably desirable and necessary to approach the full problem via simplified versions that are less demanding on computer time and storage space. Specifically, as in previous diagnostic codes, the iterations required at each time step for full self-consistency should initially be omitted in favour of approximate formulae relating gas characteristics to the local MC radiation field.

Acknowledgements. I am grateful to S. A. Sim for detailed comments on the manuscript.

\section{References}

Abbott, D. C., \& Lucy, L. B. 1985, ApJ, 288, 679

Ambwani, K., \& Sutherland, P. 1988, ApJ, 325, 820

Arnett, W. D. 1980, ApJ, 237, 541

Arnett, W. D. 1982, ApJ, 253, 785

Castor, J. I. 1972, ApJ, 178, 779

Colgate, S. A., Petschek, A. G., \& Kriese, J. T. 1980, ApJ, 237, L81

Eastman, R. G., \& Pinto, P. A. 1993, ApJ, 412, 731

Fransson, C., \& Kozma, C. 1993, ApJ, 408, L25

Harries, T. J., Monnier, J. D., Symington, N. H., \& Kurosawa, R. 2004, MNRAS, 350, 565

Höflich, P. 2002, New Astron. Rev., 46, 475

Höflich, P. 2003, in Stellar Atmosphere Modeling, ASP Conf. Proc., 28, 185

Kasen, D., Nugent, P., Thomas, R. C., \& Wang, L. 2004, ApJ, in press [arXiv: astro-ph/0311009]

Kurosawa, R., Harries, T. J., Bate, M. R., \& Symington, N. H. 2004, MNRAS, in press [arXiv: astro-ph/0403582]

Lucy, L. B. 1987, in ESO Workshop on SN 1987A, ed. I. J. Danziger, 417

Lucy, L. B. 1999a, A\&A, 344, 282

Lucy, L. B. 1999b, A\&A, 345, 211

Lucy, L. B. 2002, A\&A, 384, 725 (Paper I)

Lucy, L. B. 2003, A\&A, 403, 261 (Paper II)

Mazzali, P. A., \& Lucy, L. B. 1993, A\&A, 279, 447

Meyerott, R. E. 1978, ApJ, 221, 975

Milne, P. A., Hungerford, A. L., Fryer, C. L., et al. 2004, ApJ, in press [arXiv:astro-ph/0406173]

Niccolini, G., Woitke, P., \& Lopez, B. 2003, A\&A, 399, 703

Pinto, P. A., \& Eastman, R. G. 2000a, ApJ, 530, 744

Pinto, P. A., \& Eastman, R. G. 2000b, ApJ, 530, 757

Press, W. H., Teukolsky, S. A., Vetterling, W. T., \& Flannery, B. P. 1992, Numerical Recipes (Cambridge: Cambridge Univ. Press)

Richtmyer, R. D. 1957, Difference Methods for Initial-Value Problems (New York: Interscience Publishers)

Schwarzschild, M. 1958, Structure and Evolution of the Stars (Princeton University Press)

Springmann, U., \& Puls, J. 1998, in Boulder-Munich II: Properties of Hot, Luminous Stars, ed. I. D. Howarth, ASP Conf. Ser., 131, 286

Wheeler, J. C. 2004, in Cosmic Explosions in Three Dimensions: Asymmetries in Supernovae and Gamma-Ray Bursts, ed. P. Höflich, P. Kumar, \& J. C. Wheeler (Cambridge University Press) Wolf, S. 2003, ApJ, 582, 859

Woosley, S. E., Pinto, P. A., \& Hartmann, D. 1989, ApJ, 346, 395 